\title{
A NEW APPROACH To M(G)-Group SOFT UNION ACTION AND ITS APPLICATIONS To M(G)-Group THEORY
}

\author{
P.Jeyaraman ${ }^{1} \&$ R.Nagarajan ${ }^{2}$ \\ ${ }^{1}$ Assistant Professor, Department of Mathematics, Bharathiar University,Coimbatore-046. \\ ${ }^{2}$ Associate Professor, Department of Mathematics ,J J College of Engineering \& \\ Technology Tiruchirappalli- 620009, Tamilnadu, India
}

\section{ABSTRACT:}

In this paper, we define a new type of $M(G)$-group action, called $M(G)$-group soft union( $S U)$ action and $M(G)$-ideal soft union(SU) action on a soft set. This new concept illustrates how a soft set effects on an $M(G)$-group in the mean of union and inclusion of sets and its function as bridge among soft set theory, set theory and $M(G)$-group theory. We also obtain some analog of classical $M(G)$ - group theoretic concepts for $M(G)$-group $S U$-action. Finally, we give the application of $S U$-actions on $M(G)$-group to $M(G)$-group theory.

\section{KEYWORDS:}

soft set, $M(G)$-group, $M(G)$-group $S U$-action, $M(G)$--ideal $S U$-action, soft pre-image, soft anti-image, $\alpha$ inclusion.

Ams Mathematics SubJECt ClaSsification: 03E70,08E40,

\section{INTRODUCTION:}

Soft set theory as in $[1,2,11,14,15,16,18,25,28]$ was introduced in 1999 by Molodtsov [22] for dealing with uncertainties and it has gone through remarkably rapid strides in the mean of algebraic structures. Maji et al. [19] presented some definitions on soft sets and based on the analysis of several operations on soft sets Ali et al. [3] introduced several operations Moreover, Atagun and Sezgin [4] defined the concepts of soft sub rings and ideals of a ring, soft subfields of a field and soft sub modules of a module and studied their related properties with respect to soft set operations. Furthermore, soft set relations and functions [5] and soft mappings [21] with many related concepts were discussed. The theory of soft set has also a wide-ranging applications especially in soft decision making as in the following studies: [6, 7, 23, 29] Sezgin et.al [25] introduced a new concept to the literature of $\mathrm{N}$-group called $\mathrm{N}$-group soft intersection action. Operations of soft sets have been studied by some authors, too. of soft sets and Sezgin and Atagun [26] studied on soft set operations as well. In this paper, we define a new type of $M(G)-$ group action on a soft set, which we call $\mathrm{M}(\mathrm{G})$ - group soft union action and abbreviate as " $M(G)$ group SU action "which is based on the inclusion relation and union of sets. Since $\mathrm{M}(\mathrm{G})$ - group DOI: 10.14810/ijscmc.2017.6101 
SU-action gathers soft set theory and set theory and $\mathrm{M}(\mathrm{G})$-group theory, it is useful in improving the soft set theory with respect to $\mathrm{M}(\mathrm{G})$ - group structures. Based on this new notion, we then introduce the concepts of $\mathrm{M}(\mathrm{G})$-ideal SU-action and show that if $\mathrm{M}(\mathrm{G})$-group SU-action over $\mathrm{U}$. Moreover, we investigate these notions with respect to soft image, soft pre-image and give their applications to $\mathrm{M}(\mathrm{G})$ - group theory.

\section{PRELIMINARIES:}

In this section, we recall some basic notions relevant to $\mathrm{M}(\mathrm{G})$ - groups and soft sets. By a nearring, we shall mean an algebraic system $(\mathrm{M}(\mathrm{G}),+, \bullet)$,

where

$\left(\mathrm{N}_{1}\right)(\mathrm{M}(\mathrm{G}),+)$ forms a group (not necessarily abelian)

$\left(\mathrm{N}_{2}\right)(\mathrm{M}(\mathrm{G}), \bullet)$ forms a semi group and

$\left(\mathrm{N}_{3}\right)(\mathrm{x}+\mathrm{y}) \mathrm{z}=\mathrm{xz}+\mathrm{yz}$ for all $\mathrm{x}, \mathrm{y}, \mathrm{z} \in \mathrm{G}$.

Throughout this paper, $M(G)$ will always denote right near-ring. A normal subgroup $H$ of $M(G)$ is called a left ideal of $M(G)$ if $g(f+i)$-gf $\in H$ for all $g, f \in M(G)$ and $i \in I$ and denoted by $\mathrm{H} \triangleleft_{\ell} \mathrm{M}(\mathrm{G})$. For a near-ring $\mathrm{M}(\mathrm{G})$, the zero-symmetric part of $\mathrm{M}(\mathrm{G})$ denoted by $\mathrm{M}_{0}(\mathrm{G})$ is defined by $\mathrm{M}_{0}(\mathrm{G})=\{\mathrm{g} \in \mathrm{S} / \mathrm{g} 0=0\}$.

Let $\mathrm{G}$ be a group, written additively but not necessarily abelian, and let $\mathrm{M}(\mathrm{G})$ be the set $\{\mathrm{f} / \mathrm{f}$ : $\mathrm{G} \rightarrow \mathrm{G}\}$ of all functions from $\mathrm{G}$ to $\mathrm{G}$. An addition operation can be defined on $\mathrm{M}(\mathrm{G})$; given $\mathrm{f}, \mathrm{g}$ in $M(G)$, then the mapping $f+g$ from $G$ to $G$ is given by $(f+g) x=f(x)+g(x)$ for $x$ in G.Then $(\mathrm{M}(\mathrm{G}),+)$ is also group, which is abelian if and only if $\mathrm{G}$ is abelian. Taking the composition of mappings as the product, $\mathrm{M}(\mathrm{G})$ becomes a near-ring.

Let $\mathrm{G}$ be a group. Then, under the operation below;

$\mu: \mathrm{M}(\mathrm{G}) \times \mathrm{G} \rightarrow \mathrm{G}$

$(f, a) \rightarrow$ fa

$(\mathrm{G}, \mu)$ is called $\mathrm{M}(\mathrm{G})$-group. Let $\mathrm{M}(\mathrm{G})$ be a near-ring, $\mathrm{G}_{1}$ and $\mathrm{G} 2$ two $\mathrm{M}(\mathrm{G})$-groups. Then $\phi: \mathrm{G}_{1}$ $\rightarrow \mathrm{G}_{2}$ is called $\mathrm{M}(\mathrm{G})$ - homomorphism if for all $\mathrm{x}, \mathrm{y} \varepsilon \mathrm{G}_{1}$, for all $\mathrm{g} \varepsilon \mathrm{M}(\mathrm{G})$,

(i) $\quad \Phi(\mathrm{x}+\mathrm{y})=\phi(\mathrm{x})+\phi(\mathrm{y})$

(ii) $\Phi(\mathrm{gx})=\mathrm{g} \phi(\mathrm{x})$. It is denoted by G. Clearly $\mathrm{M}(\mathrm{G})$ itself is an $\mathrm{M}(\mathrm{G})$-group by natural operations.

For all undefined concepts and notions we refer to (24). From now on, $U$ refers to on initial universe, $\mathrm{E}$ is a set of parameters $\mathrm{P}(\mathrm{U})$ is the power set of $\mathrm{U}$ and $\mathrm{A}, \mathrm{B}, \mathrm{C} \subseteq \mathrm{E}$.

2.1.Definition[22]: A pair $(F, A)$ is called a soft set over $U$, where $F$ is a mapping given by $\mathrm{F}: \mathrm{A} \rightarrow \mathrm{P}(\mathrm{U})$.

In other words, a soft set over $\mathrm{U}$ is a parameterized family of subsets of the universe $\mathrm{U}$.

Note that a soft set $(\mathrm{F}, \mathrm{A})$ can be denoted by $\mathrm{F}_{\mathrm{A}}$. In this case, when we define more than one soft set in some subsets $A, B, C$ of parameters $E$, the soft sets will be denoted by $F_{A}, F_{B}, F_{C}$, respectively. On the other case, when we define more than one soft set in a subset A of the set of 
parameters $\mathrm{E}$, the soft sets will be denoted by $\mathrm{F}_{\mathrm{A}}, \mathrm{G}_{\mathrm{A}}, \mathrm{H}_{\mathrm{A}}$, respectively. For more details, we refer to $[11,17,18,26,29,7]$.

2.2.Definition[6] :The relative complement of the soft set $F_{A}$ over $U$ is denoted by $F_{A}^{r}$, where $\mathrm{F}_{\mathrm{A}}^{\mathrm{r}}: \mathrm{A} \rightarrow \mathrm{P}(\mathrm{U})$ is a mapping given as $\mathrm{F}_{\mathrm{A}}^{\mathrm{r}}(\mathrm{a})=\mathrm{U} \backslash \mathrm{F}_{\mathrm{A}}(\mathrm{a})$, for all $\mathrm{a} \in \mathrm{A}$.

2.3.Definition[6]: Let $F_{A}$ and $G_{B}$ be two soft sets over $U$ such that $A \cap B \neq \varnothing$,. The restricted intersection of $F_{A}$ and $G_{B}$ is denoted by $F_{A} \in G_{B}$, and is defined as $F_{A} \in G_{B}=(H, C)$, where $\mathrm{C}=\mathrm{A} \cap \mathrm{B}$ and for all $\mathrm{c} \in \mathrm{C}, \mathrm{H}(\mathrm{c})=\mathrm{F}(\mathrm{c}) \cap \mathrm{G}(\mathrm{c})$.

2.4.Definition[6]: Let $F_{A}$ and $G_{B}$ be two soft sets over $U$ such that $A \cap B \neq \emptyset$,. The restricted union of $F_{A}$ and $G_{B}$ is denoted by $F_{A} U_{R} G_{B}$, and is defined as $F_{A} U_{R} G_{B}=(H, C)$, where $C=A \cap B$ and for all $\mathrm{c} \in \mathrm{C}, \mathrm{H}(\mathrm{c})=\mathrm{F}(\mathrm{c}) \cup \mathrm{G}(\mathrm{c})$.

2.5 Definition[12]: Let $\mathrm{F}_{\mathrm{A}}$ and $\mathrm{G}_{\mathrm{B}}$ be soft sets over the common universe $\mathrm{U}$ and $\psi$ be a function from $\mathrm{A}$ to $\mathrm{B}$. Then we can define the soft set $\psi\left(\mathrm{F}_{\mathrm{A}}\right)$ over $\mathrm{U}$, where $\psi\left(\mathrm{F}_{\mathrm{A}}\right): \mathrm{B} \rightarrow \mathrm{P}(\mathrm{U})$ is a set valued function defined by $\psi\left(\mathrm{F}_{\mathrm{A}}\right)(\mathrm{b})=\mathrm{U}\{\mathrm{F}(\mathrm{a}) \mid \mathrm{a} \in \mathrm{A}$ and $\psi(\mathrm{a})=\mathrm{b}\}$,

if $\psi^{-1}(\mathrm{~b}) \neq \emptyset,=0$ otherwise for all $\mathrm{b} \in \mathrm{B}$. Here, $\psi\left(\mathrm{F}_{\mathrm{A}}\right)$ is called the soft image of $\mathrm{F}_{\mathrm{A}}$ under $\psi$. Moreover we can define a soft set $\psi^{-1}\left(\mathrm{G}_{\mathrm{B}}\right)$ over $\mathrm{U}$, where $\psi^{-1}\left(\mathrm{G}_{\mathrm{B}}\right): \mathrm{A} \rightarrow \mathrm{P}(\mathrm{U})$ is a set-valued function defined by $\psi^{-1}\left(\mathrm{G}_{\mathrm{B}}\right)(\mathrm{a})=\mathrm{G}(\psi(\mathrm{a}))$ for all $\mathrm{a} \in \mathrm{A}$. Then, $\psi^{-1}\left(\mathrm{G}_{\mathrm{B}}\right)$ is called the soft pre image (or inverse image) of $\mathrm{G}_{\mathrm{B}}$ under $\psi$.

2.6.Definition[13]: Let $F_{A}$ and $G_{B}$ be soft sets over the common universe $U$ and $\psi$ be a function from $\mathrm{A}$ to $\mathrm{B}$. Then we can define the soft set $\psi^{\star}\left(\mathrm{F}_{\mathrm{A}}\right)$ over $\mathrm{U}$, where $\psi^{\star}\left(\mathrm{F}_{\mathrm{A}}\right): \mathrm{B} \rightarrow \mathrm{P}(\mathrm{U})$ is a set-valued function defined by $\psi^{\star}\left(\mathrm{F}_{\mathrm{A}}\right)(\mathrm{b})=\cap\{\mathrm{F}(\mathrm{a}) \mid \mathrm{a} \in \mathrm{A}$ and $\psi(\mathrm{a})=\mathrm{b}\}$, if $\psi^{-1}(\mathrm{~b}) \neq \emptyset$,

$=0$ otherwise for all $\mathrm{b} \in \mathrm{B}$. Here, $\psi^{\star}\left(\mathrm{F}_{\mathrm{A}}\right)$ is called the soft anti image of $\mathrm{F}_{\mathrm{A}}$ under $\psi$.

2.7 Definition [8]: Let $f_{A}$ be a soft set over $U$ and $\alpha$ be a subset of $U$. Then, lower $\alpha$-inclusion of a soft set $\mathrm{f}_{\mathrm{A}}$, denoted by $\mathrm{f}^{\alpha}{ }_{\mathrm{A}}$, is defined as $\mathrm{f}^{\alpha}{ }_{\mathrm{A}}=\left\{\mathrm{x} \in \mathrm{A}: \mathrm{f}_{\mathrm{A}}(\mathrm{x}) \subseteq \alpha\right\}$

\section{M(G) -GrouP SU-ACTION}

In this section, we first define $\mathrm{M}(\mathrm{G})$-group soft union action, abbreviated as $\mathrm{M}(\mathrm{G})$-group SUaction with illustrative examples. We then study their basic results with respect to soft set operation.

3.1Definition: Let $\mathrm{S}$ be an $\mathrm{M}(\mathrm{G})$ - group and $f_{s}$ be a fuzzy soft set over $\mathrm{U}$, then $f_{s}$ is called fuzzy $\mathrm{SU}$-action on $\mathrm{M}(\mathrm{G})$ - group over $\mathrm{U}$ if it satisfies the following conditions;

$\left(\mathrm{FS}_{\mathrm{U}} \mathrm{N}-1\right) f_{s}(\mathrm{x}+\mathrm{y}) \subseteq f_{s}(\mathrm{x}) \mathrm{U} f_{s}(\mathrm{y})$

$\left(\mathrm{FS}_{\mathrm{U}} \mathrm{N}-2\right) f_{S}(-\mathrm{x}) \subseteq f_{S}(\mathrm{x})$

$\left(\mathrm{FS}_{\mathrm{U}} \mathrm{N}-3\right) f_{s}(\mathrm{gx}) \subseteq f_{s}(\mathrm{x})$

For all $\mathrm{x}, \mathrm{y} \in \mathrm{S}$ and $\mathrm{g} \in \mathrm{M}(\mathrm{G})$. 
International Journal of Soft Computing, Mathematics and Control (IJSCMC), Vol. 6, No.1, February 2017

3.1Example: Consider the near-ring module $M(G)=\{e, f, g, h\}$, be the near-ring under the operation defined by the following table:

\begin{tabular}{|c|cccc|}
\hline+ & e & f & g & h \\
\hline e & e & f & g & h \\
f & f & e & f+g & f+h \\
g & g & g+h & e & g+h \\
h & h & h+f & h+g & e \\
& & & & \\
& & & & \\
\hline
\end{tabular}

\begin{tabular}{|l|llcc|}
\hline$\cdot$ & e & f & g & h \\
\hline 0 & e & f & g & h \\
f & f & e & f $\bullet$ & f $\bullet$ h \\
g & g & g $\bullet$ h & e & g $\bullet$ h \\
h & h & h $\bullet$ f & $h \bullet g$ & e \\
& & & & \\
& & & & \\
\hline
\end{tabular}

Let $\mathrm{G}=\mathrm{M}(\mathrm{G})$ be the set of functions and

and $U=\left\{\left[\begin{array}{ll}f & e \\ f & e\end{array}\right] / f, e \in M(G)\right\}, 2 \times 2$ matrices with four terms, is the universal set .we construct a soft set.

$f_{s}(\mathrm{e})=\left\{\left[\begin{array}{ll}e & 0 \\ e & 0\end{array}\right],\right\}, \quad f_{s}(\mathrm{f})=f_{s}(\mathrm{~g})=f_{s}(\mathrm{~h})=\left\{\left[\begin{array}{ll}f & 0 \\ f & 0\end{array}\right],\left[\begin{array}{ll}g & 0 \\ g & 0\end{array}\right],\left[\begin{array}{ll}3 & 0 \\ 0 & 0\end{array}\right]\right\}$,

Then one can easily show that the soft set $f_{s}$ is a $\mathrm{M}(\mathrm{G})$-group SU-action over $\mathrm{U}$.

3.1 Proposition: Let $f_{s}$ be a fuzzy SU-action on $\mathrm{M}(\mathrm{G})$ - group over $\mathrm{U}$. Then, $f_{s}(0) \subseteq f_{s}(\mathrm{x})$ for all $\mathrm{x} \in S$.

Proof: Assume that $f_{S}$ is fuzzy SU-action over U.Then, for all $\mathrm{x} \in \mathrm{S}$, $f_{s}(0)=f_{s}(\mathrm{x}-\mathrm{x}) \subseteq f_{s}(\mathrm{x}) \cup f_{s}(-\mathrm{x})=f_{s}(\mathrm{x}) \cup f_{s}(\mathrm{x})=f_{s}(\mathrm{x})$.

3.1 Theorem: Let $\mathrm{S}$ be a fuzzy SU-action on $\mathrm{M}(\mathrm{G})$ - group and $f_{S}$ be a fuzzy soft set over $\mathrm{U}$. Then $f_{s}$ is $\mathrm{SU}$-action of $\mathrm{M}(\mathrm{G})$ - group over $\mathrm{U}$ if and only if

(i) $f_{S}(\mathrm{x}-\mathrm{y}) \subseteq f_{S}(\mathrm{x}) \cup f_{s}(\mathrm{y})$

(ii) $f_{S}(\mathrm{gx}) \subseteq f_{S}(\mathrm{x})$ for all $\mathrm{x}, \mathrm{y} \in \mathrm{S}$ and $\mathrm{g} \in \mathrm{M}(\mathrm{G})$.

Proof: Suppose $f_{S}$ is a fuzzy SU-action on $\mathrm{M}(\mathrm{G})$ - group over $\mathrm{U}$. Then, by definition-3.1, $f_{s}(\mathrm{xy}) \subseteq f_{S}(\mathrm{y})$ and $f_{s}(\mathrm{x}-\mathrm{y}) \subseteq f_{S}(\mathrm{x}) \cup f_{s}(-\mathrm{y})=f_{s}(\mathrm{x}) \cup f_{s}(\mathrm{y})$ for all $\mathrm{x}, \mathrm{y} \in \mathrm{S}$

Conversely, assume that $f_{s}(\mathrm{xy}) \subseteq f_{S}(\mathrm{y})$ and $f_{S}(\mathrm{x}-\mathrm{y}) \subseteq f_{S}(\mathrm{x}) \cup f_{s}(\mathrm{y})$ for all $\mathrm{x}, \mathrm{y} \in \mathrm{S}$.

If we choose $\mathrm{x}=0$, then $f_{s}(0-\mathrm{y})=f_{s}(-\mathrm{y}) \subseteq f_{s}(0) \cup f_{s}(\mathrm{y})=f_{s}(\mathrm{y})$ by proposition-3.1. Similarly

$f_{s}(\mathrm{y})=f_{S}(-(-\mathrm{y})) \subseteq f_{S}(-\mathrm{y})$,thus $f_{s}(-\mathrm{y})=f_{s}(\mathrm{y})$ for all $\mathrm{y} \in \mathrm{S}$. Also, by assumption $f_{s}(\mathrm{x}-\mathrm{y}) \subseteq f_{s}(\mathrm{x}) \cup f_{s}(-\mathrm{y})=f_{s}(\mathrm{x}) \cup f_{s}(\mathrm{y})$. This complete the proof.

3.2Theorem: Let $f_{s}$ be a fuzzy $\mathrm{SU}$-action on $\mathrm{M}(\mathrm{G})$ - group over $\mathrm{U}$.

(i) If $f_{S}(\mathrm{x}-\mathrm{y})=f_{S}(0)$ for any $\mathrm{x}, \mathrm{y} \in \mathrm{S}$, then $f_{S}(\mathrm{x})=f_{S}(\mathrm{y})$.

(ii) $f_{S}(\mathrm{x}-\mathrm{y})=f_{S}(0)$ for any $\mathrm{x}, \mathrm{y} \in \mathrm{S}$, then $f_{S}(\mathrm{x})=f_{S}(\mathrm{y})$. 
Proof: Assume that $f_{S}(\mathrm{x}-\mathrm{y})=f_{S}(0)$ for any $\mathrm{x}, \mathrm{y} \in \mathrm{S}$, then

$$
\begin{aligned}
f_{s}(\mathrm{x}) & =f_{s}(\mathrm{x}-\mathrm{y}+\mathrm{y}) \subseteq f_{s}(\mathrm{x}-\mathrm{y}) \cup f_{s}(\mathrm{y}) \\
& =f_{s}(0) \cup f_{s}(\mathrm{y})=f_{s}(\mathrm{y})
\end{aligned}
$$

and similarly,

$$
\begin{aligned}
f_{s}(\mathrm{y}) & =f_{s}((\mathrm{y}-\mathrm{x})+\mathrm{x}) \subseteq f_{s}(\mathrm{y}-\mathrm{x}) \cup f_{s}(\mathrm{x}) \\
& =f_{s}(-(\mathrm{y}-\mathrm{x})) \cup f_{s}(\mathrm{x}) \\
& =f_{s}(0) \cup f_{s}(\mathrm{x})=f_{s}(\mathrm{x})
\end{aligned}
$$

Thus, $f_{s}(\mathrm{x})=f_{s}(\mathrm{y})$ which completes the proof .Similarly, we can show the result (ii).

It is known that if $S$ is an $M(G)$ - group, then $(S,+)$ is a group but not necessarily abelian.

That is, for any $\mathrm{x}, \mathrm{y} \in \mathrm{S}, \mathrm{x}+\mathrm{y}$ needs not be equal to $\mathrm{y}+\mathrm{x}$. However, we have the following:

3.3Theorem: Let $f_{S}$ be fuzzy $\mathrm{SU}$-action on $\mathrm{M}(\mathrm{G})$ - group over $\mathrm{U}$ and $\mathrm{x} \in \mathrm{S}$. Then,

$$
f_{S}(\mathrm{x})=f_{S}(0) \Leftrightarrow f_{S}(\mathrm{x}+\mathrm{y})=f_{S}(\mathrm{y}+\mathrm{x})=f_{S}(\mathrm{y}) \text { for all } \mathrm{y} \in \mathrm{S} \text {. }
$$

Proof: Suppose that $f_{S}(\mathrm{x}+\mathrm{y})=f_{S}(\mathrm{y}+\mathrm{x})=f_{S}(\mathrm{y})$ for all $\mathrm{y} \in \mathrm{S}$. Then, by choosing $\mathrm{y}=0$, we obtain that $f_{s}(\mathrm{x})=f_{s}(0)$.

Conversely, assume that $f_{S}(\mathrm{x})=f_{S}(0)$. Then by proposition-3.1, we have

$$
f_{s}(0)=f_{s}(\mathrm{x}) \subset f_{s}(\mathrm{y}), \forall \mathrm{y} \in \mathrm{S} \text {. }
$$

Since $f_{S}$ is fuzzy SU-action on N-module over $\mathrm{U}$, then

$$
\begin{aligned}
& f_{s}(\mathrm{x}+\mathrm{y}) \subseteq f_{s}(\mathrm{x}) \cup f_{s}(\mathrm{y})=f_{s}(\mathrm{y}), \forall \mathrm{y} \in \mathrm{S} . \text { Moreover, for all } \mathrm{y} \in \mathrm{S} \\
& \begin{aligned}
f_{S}(\mathrm{y}) & \left.=f_{S}((-\mathrm{x})+\mathrm{x})+\mathrm{y}\right)=f_{s}(-\mathrm{x}+(\mathrm{x}+\mathrm{y})) \subseteq f_{s}(-\mathrm{x}) \cup f_{s}(\mathrm{x}+\mathrm{y}) \\
& =f_{S}(\mathrm{x}) \cup f_{s}(\mathrm{x}+\mathrm{y})=f_{S}(\mathrm{x}+\mathrm{y})
\end{aligned}
\end{aligned}
$$

Since by equation $(1), f_{S}(\mathrm{x}) \subseteq f_{S}(\mathrm{y})$ for all $\mathrm{y} \in \mathrm{S}$ and $\mathrm{x}, \mathrm{y} \in \mathrm{S}$,implies that $\mathrm{x}+\mathrm{y} \in \mathrm{S}$.Thus, it follows that $f_{S}(\mathrm{x}) \subseteq f_{S}(\mathrm{x}+\mathrm{y})$. So $f_{S}(\mathrm{x}+\mathrm{y})=f_{s}(\mathrm{y})$ for all $\mathrm{y} \in \mathrm{S}$.

Now, let $\mathrm{x} \in \mathrm{S}$. Then, for all $\mathrm{x}, \mathrm{y} \in \mathrm{S}$

$$
\begin{aligned}
f_{s}(\mathrm{y}+\mathrm{x}) & =f_{s}(\mathrm{y}+\mathrm{x}+(\mathrm{y}-\mathrm{y})) \\
& =f_{s}(\mathrm{y}+(\mathrm{x}+\mathrm{y})-\mathrm{y}) \\
& \subseteq f_{s}(\mathrm{y}) \cup f_{s}(\mathrm{x}+\mathrm{y}) \cup f_{s}(\mathrm{y}) \\
& =f_{s}(\mathrm{y}) \cup f_{s}(\mathrm{x}+\mathrm{y})=f_{s}(\mathrm{y})
\end{aligned}
$$

Since $f_{S}(\mathrm{x}+\mathrm{y})=f_{s}(\mathrm{y})$. Furthermore, for all $\mathrm{y} \in \mathrm{S}$

$$
\begin{aligned}
f_{s}(\mathrm{y}) & =f_{s}(\mathrm{y}+(\mathrm{x}-\mathrm{x})) \\
& =f_{s}((\mathrm{y}+\mathrm{x})-\mathrm{x}) \\
& \subseteq f_{s}(\mathrm{y}+\mathrm{x}) \cup f_{s}(\mathrm{x}) \\
& =f_{s}(\mathrm{y}+\mathrm{x}) \text { by equation }(1)
\end{aligned}
$$

It follows that $f_{S}(\mathrm{y}+\mathrm{x})=f_{s}(\mathrm{y})$ and so $f_{S}(\mathrm{x}+\mathrm{y})=f_{S}(\mathrm{y}+\mathrm{x})=f_{s}(\mathrm{y})$, for all $\mathrm{y} \in \mathrm{S}$, which completes the proof.

3.4Theorem: Let $\mathrm{S}$ be a near-field and $f_{S}$ be a fuzzy soft set over $\mathrm{U}$. If $f_{S}(0) \subseteq f_{S}(1)=f_{S}(\mathrm{x})$ for all $0 \neq x \in S$, then it is fuzzy $S U$-action on $M(G)$ - group over $U$. 
Proof: Suppose that $f_{S}(0) \subseteq f_{S}(1)=f_{S}(\mathrm{x})$ for all $0 \neq \mathrm{x} \in \mathrm{S}$. In order to prove that it is fuzzy SU-action on $\mathrm{M}(\mathrm{G})$ - group over $\mathrm{U}$, it is enough to prove that $f_{s}(\mathrm{x}-\mathrm{y}) \subseteq f_{s}(\mathrm{x}) \cup f_{s}(\mathrm{y})$ and $f_{s}(\mathrm{gx}) \subseteq f_{s}(\mathrm{x})$.

Let $x, y \in S$. Then we have the following cases:

Case-1: Suppose that $\mathrm{x} \neq 0$ and $\mathrm{y}=0$ or $\mathrm{x}=0$ and $\mathrm{y} \neq 0$. Since $\mathrm{S}$ is a near-field, so it follows that $\mathrm{gx}=0$ and $f_{S}(\mathrm{gx})=f_{S}(0)$. since $f_{S}(0) \subseteq f_{S}(\mathrm{x})$, for all $\mathrm{x} \in \mathrm{S}, \mathrm{so} f_{S}(\mathrm{nx})=f_{S}(0) \subseteq f_{S}(\mathrm{x})$, and $f_{s}(\mathrm{nx})=f_{s}(0) \subseteq f_{s}(\mathrm{y})$. This imply $f_{s}(\mathrm{gx}) \subseteq f_{s}(\mathrm{x})$.

Case-2: Suppose that $\mathrm{x} \neq 0$ and $\mathrm{y} \neq 0$. It follows that $\mathrm{nx} \neq 0$.Then, $f_{S}(\mathrm{nx})=f_{S}(1)=f_{S}(\mathrm{x})$ and $f_{s}(\mathrm{gx})=f_{s}(1)=f_{s}(\mathrm{y})$, which implies that $f_{s}(\mathrm{gx}) \subseteq f_{s}(\mathrm{x})$.

Case-3: suppose that $\mathrm{x}=0$ and $\mathrm{y}=0$, then clearly $f_{S}(\mathrm{gx}) \subseteq f_{s}(\mathrm{x})$. Hence $f_{s}(\mathrm{gx}) \subseteq f_{S}(\mathrm{x})$, for all $\mathrm{x}, \mathrm{y} \in \mathrm{S}$.

Now, let $\mathrm{x}, \mathrm{y} \in \mathrm{S}$. Then $\mathrm{x}-\mathrm{y}=0$ or $\mathrm{x}-\mathrm{y} \neq 0$. If $\mathrm{x}-\mathrm{y}=0$, then either $\mathrm{x}=\mathrm{y}=0$ or $\mathrm{x} \neq 0, \mathrm{y} \neq 0$ and $\mathrm{x}=\mathrm{y}$. But, since $f_{s}(\mathrm{x}-\mathrm{y})=f_{s}(0) \subseteq f_{S}(\mathrm{x})$, for all $\mathrm{x} \in \mathrm{S}$, it follows that $f_{s}(\mathrm{x}-\mathrm{y})=f_{S}(0) \subseteq f_{s}(\mathrm{x}) \cup f_{s}(\mathrm{y})$. If $\mathrm{x}-\mathrm{y} \neq 0$, then either $\mathrm{x} \neq 0, \mathrm{y} \neq 0$ and $\mathrm{x} \neq \mathrm{y}$ or $\mathrm{x} \neq 0$ and $\mathrm{y}=0$ or $\mathrm{x}=0$ and $\mathrm{y} \neq 0$.

Assume that $\mathrm{x} \neq 0, \mathrm{y} \neq 0$ and $\mathrm{x} \neq \mathrm{y}$. This follows that

$$
f_{s}(\mathrm{x}-\mathrm{y})=f_{s}(1)=f_{s}(\mathrm{x}) \subseteq f_{s}(\mathrm{x}) \cup f_{s}(\mathrm{y}) .
$$

Now, let $\mathrm{x} \neq 0$ and $\mathrm{y}=0$. Then $f_{S}(\mathrm{x}-\mathrm{y}) \subseteq f_{S}(\mathrm{x}) \cup f_{S}(\mathrm{y})$. Finally, let $\mathrm{x}=0$ and $\mathrm{y} \neq 0$.

Then, $f_{s}(\mathrm{x}-\mathrm{y}) \subseteq f_{s}(\mathrm{x}) \cup f_{s}(\mathrm{y})$. Hence $f_{s}(\mathrm{x}-\mathrm{y}) \subseteq f_{s}(\mathrm{x}) \cup f_{s}(\mathrm{y})$, for all $\mathrm{x}, \mathrm{y} \in \mathrm{S}$.

Thus, $f_{s}$ is fuzzy SU-action on $\mathrm{M}(\mathrm{G})$ - group over $\mathrm{U}$.

3.5Theorem: Let $f_{S}$ and $f_{T}$ be two fuzzy SU-action on $\mathrm{M}(\mathrm{G})$ - group over $\mathrm{U}$. Then $f_{S} \wedge f_{T}$ is fuzzy soft SU-action on $\mathrm{M}(\mathrm{G})$ - group over $\mathrm{U}$.

Proof: let $\left(x_{1}, y_{1}\right),\left(x_{2}, y_{2}\right) \in \mathrm{S} \times \mathrm{T}$.Then

$$
\begin{aligned}
f_{S \wedge T}\left(\left(x_{1}, y_{1}\right)-\left(x_{2}, y_{2}\right)\right) & =f_{S \wedge T}\left(x_{1-} x_{2}, y_{1-} y_{2}\right) \\
& =f_{S}\left(x_{1-} x_{2}\right) \cap f_{T}\left(y_{1-} y_{2}\right) \\
& \subseteq\left(f_{S}\left(x_{1}\right) \cup f_{S}\left(x_{2}\right)\right) \cap\left(f_{T}\left(y_{1}\right) \cup f_{T}\left(y_{2}\right)\right) \\
& =\left(f_{S}\left(x_{1}\right) \cup f_{T}\left(y_{1}\right)\right) \cap\left(f_{S}\left(x_{2}\right) \cup f_{T}\left(y_{2}\right)\right) \\
& =f_{S \wedge T}\left(x_{1}, y_{1}\right) \cap f_{S \wedge T}\left(x_{2}, y_{2}\right)
\end{aligned}
$$

and

$$
\begin{aligned}
f_{S \wedge T}\left(\left(g_{1}, g_{2}\right),\left(x_{2}, y_{2}\right)\right) & =f_{S \wedge T}\left(n_{1} x_{2}, n_{2} y_{2}\right) \\
& =f_{S}\left(g_{1} x_{2}\right) \cap f_{T}\left(g_{2} y_{2}\right) \\
& \subseteq f_{S}\left(x_{2}\right) \cap f_{T}\left(y_{2}\right) \\
& =f_{S \wedge T}\left(x_{2}, y_{2}\right)
\end{aligned}
$$

Thus $f_{S} \wedge f_{T}$ is fuzzy SU-action on $\mathrm{M}(\mathrm{G})$ - group over $\mathrm{U}$.

Note that $f_{S} \vee f_{T}$ is not fuzzy SU-action on $\mathrm{M}(\mathrm{G})$ - group over $\mathrm{U}$. 
3.2Example: Assume $\mathrm{U}=p_{3}$ is the universal set. Let $\mathrm{S}=Z_{3}$ and $\mathrm{H}=\left\{\left[\begin{array}{ll}a & a \\ b & b\end{array}\right] / a, b \in Z_{3}\right\}$ $2 \times 2$ matrices with $Z_{3}$ terms, be set of parameters. We define fuzzy SU-action on $\mathrm{M}(\mathrm{G})$ - group $f_{S}$ over $\mathrm{U}=p_{3}$ by

$f_{S}(0)=p_{3}$

$f_{S}(1)=\left\{(1),\left(\begin{array}{lll}1 & 2\end{array}\right),\left(\begin{array}{lll}1 & 3 & 2\end{array}\right)\right\}$

$f_{S}(2)=\left\{\left(\begin{array}{lll}1\end{array}\right),\left(\begin{array}{lll}1 & 2\end{array}\right),\left(\begin{array}{lll}1 & 2 & 3\end{array}\right),\left(\begin{array}{lll}1 & 3 & 2\end{array}\right)\right\}$

We define fuzzy SU-action on $\mathrm{N}$-module $f_{H}$ over $\mathrm{U}=p_{3}$ by

$f_{H}\left\{\left[\begin{array}{ll}0 & 0 \\ 0 & 0\end{array}\right]\right\}=p_{3}$

$f_{H}\left\{\left[\begin{array}{ll}0 & 0 \\ 1 & 1\end{array}\right]\right\}=\left\{\left(\begin{array}{lll}1\end{array}\right),\left(\begin{array}{lll}1 & 2\end{array}\right),\left(\begin{array}{lll}1 & 3 & 2\end{array}\right)\right\}$

Then $f_{s} \vee f_{T}$ is not fuzzy SU-action on $\mathrm{M}(\mathrm{G})$ - group over $\mathrm{U}$.

3.2Definition: Let $f_{S}, g_{T}$ be fuzzy SU-action on $\mathrm{M}(\mathrm{G})$ - group over $\mathrm{U}$. Then product of fuzzy

SU-action on $\mathrm{M}(\mathrm{G})$ - group $f_{S}$ and $g_{T}$ is defined as $f_{S} \times g_{T}=h_{\mathrm{S} \times \mathrm{T}}$, where $h_{\mathrm{S} \times \mathrm{T}}(x, y)=f_{S}(x) \times g_{T}(\mathrm{y})$ for all $(x, y) \in \mathrm{S} \times \mathrm{T}$.

3.6Theorem: If $f_{S}$ and $g_{T}$ are fuzzy SU-action on $\mathrm{M}(\mathrm{G})$ - group over $\mathrm{U}$. Then so is $f_{S} \times g_{T}$ over $\mathrm{U} \times \mathrm{U}$.

Proof: By definition-3.2, let $f_{S} \times g_{T}=h_{\mathrm{S} \times \mathrm{T}}$, where $h_{\mathrm{S} \times \mathrm{T}}(x, y)=f_{S}(x) \times g_{T}(\mathrm{y})$ for all $(x, y) \in \mathrm{S} \times \mathrm{T}$. Then for all $\left(x_{1}, y_{1}\right),\left(x_{2}, y_{2}\right) \in \mathrm{S} \times \mathrm{T}$ and $\left(n_{1}, n_{2}\right)=\mathrm{N} \times \mathrm{N}$.

$$
\begin{aligned}
h_{\mathrm{S} \times \mathrm{T}}\left(\left(x_{1}, y_{1}\right)-\left(x_{2}, y_{2}\right)\right) & =h_{\mathrm{S} \times \mathrm{T}}\left(x_{1-} x_{2,}, y_{1-} y_{2}\right) \\
& =f_{S}\left(x_{1-} x_{2}\right) \times g_{T}\left(y_{1-} y_{2}\right) \\
& \subseteq\left(f_{S}\left(x_{1}\right) \cup f_{S}\left(x_{2}\right)\right) \times\left(g_{T}\left(y_{1}\right) \cup g_{T}\left(y_{2}\right)\right) \\
& =\left(f_{S}\left(x_{1}\right) \times g_{T}\left(y_{1}\right)\right) \cup\left(f_{S}\left(x_{2}\right) \times g_{T}\left(y_{2}\right)\right) \\
& =h_{\mathrm{S} \times \mathrm{T}}\left(x_{1}, y_{1}\right) \cup h_{\mathrm{S} \times \mathrm{T}}\left(x_{2}, y_{2}\right) \\
h_{\mathrm{S} \times \mathrm{T}}\left(\left(g_{1,} g_{2}\right)\left(x_{2}, y_{2}\right)\right) & =h_{\mathrm{S} \times \mathrm{T}}\left(n_{1} x_{2}, n_{2} y_{2}\right) \\
& =f_{S}\left(g_{1} x_{2}\right) \times g_{T}\left(g_{2} y_{2}\right) \\
& \subseteq f_{S}\left(x_{2}\right) \times g_{T}\left(y_{2}\right) \\
& =h_{\mathrm{S} \times \mathrm{T}}\left(x_{2,} y_{2}\right)
\end{aligned}
$$

Hence $f_{S} \times g_{T}=h_{\mathrm{S} \times \mathrm{T}}$ is fuzzy SU-action on $\mathrm{M}(\mathrm{G})$ - group over $\mathrm{U}$.

3.7Theorem: If $f_{S}$ and $h_{\mathrm{S}}$ are fuzzy SU-action on $\mathrm{M}(\mathrm{G})$-group over $\mathrm{U}$,then so is $f_{S} \widetilde{\cap} h_{\mathrm{S}}$ over $\mathrm{U}$.

Let $x, y \in \mathrm{s}$ and $\mathrm{n} \in \mathrm{N}$ then

$$
\begin{aligned}
\left(f_{S} \widetilde{\cap} h_{\mathrm{S}}\right)(x-y) & =f_{S}(x-y) \cap h_{\mathrm{S}}(x-y) \\
& \subseteq\left(f_{S}(x) \cup f_{S}(y)\right) \cap\left(h_{S}(x) \cup h_{S}(y)\right) \\
& =\left(f_{S}(x) \cap h_{S}(x)\right) \cup\left(f_{S}(y) \cap h_{S}(y)\right) \\
& =\left(f_{S} \widetilde{\cap} h_{\mathrm{S}}\right)(x) \cup\left(f_{S} \widetilde{\cap} h_{\mathrm{S}}\right)(y)
\end{aligned}
$$


International Journal of Soft Computing, Mathematics and Control (IJSCMC), Vol. 6, No.1, February 2017

$\left(f_{S} \widetilde{\cap} h_{\mathrm{S}}\right)(n \mathrm{x})=f_{S}(n x) \cap h_{\mathrm{S}}(n x) \subseteq f_{S}(x) \cap h_{\mathrm{S}}(x)=\left(f_{S} \widetilde{\cap} h_{\mathrm{S}}\right)(\mathrm{x})$

Therefore, $\left(f_{S} \widetilde{\cap} h_{S}\right)$ is fuzzy SU-action on $\mathrm{M}(\mathrm{G})$ - group over $\mathrm{U}$.

\section{Su-ACTION ON M(G)-IDEAL STRUCTURES}

4.1 Definition : Let $\mathrm{S}$ be an $\mathrm{M}(\mathrm{G})$ - group and $f_{S}$ be a fuzzy soft set over $\mathrm{U}$. Then $f_{S}$ is called fuzzy SU-action on $\mathrm{M}(\mathrm{G})$-ideal of $\mathrm{S}$ over $\mathrm{U}$ if the following conditions are satisfied:

(i) $\quad f_{s}(x+y) \subseteq f_{s}(x) \cup f_{s}(y)$

(ii) $\quad f_{s}(-x)=f_{s}(x)$

(iii) $\quad f_{s}(x+y-x) \subseteq f_{s}(y)$

(iv) $\quad f_{s}(g(x+y)-g x) \subseteq f_{s}(y)$ for all $x, y \in \mathrm{S}$ and $g \in \mathrm{M}(\mathrm{G})$.

Here, note that

$f_{s}(x+y) \subseteq f_{s}(x) \cup f_{s}(y)$ and $f_{s}(-x)=f_{s}(x)$ imply $f_{s}(x-y) \subseteq f_{s}(x) \cup f_{s}(y)$

4.1Example: Consider $M(G)=\{0, x, y, z\}$ with the following tables

\begin{tabular}{|c|cccc|}
\hline+ & 0 & $x$ & $y$ & $z$ \\
\hline 0 & 0 & $x$ & $y$ & $z$ \\
$x$ & $x$ & 0 & $z$ & $y$ \\
$y$ & $y$ & $z$ & 0 & $x$ \\
$z$ & $z$ & $y$ & $x$ & 0 \\
& & & & \\
\hline
\end{tabular}

\begin{tabular}{|l|llll|}
\hline. & 0 & $\mathrm{x}$ & $\mathrm{y}$ & $\mathrm{z}$ \\
\hline 0 & 0 & 0 & 0 & 0 \\
$\mathrm{x}$ & 0 & 0 & 0 & $\mathrm{x}$ \\
$\mathrm{y}$ & 0 & $\mathrm{x}$ & $\mathrm{y}$ & $\mathrm{y}$ \\
$\mathrm{z}$ & 0 & $\mathrm{x}$ & $\mathrm{y}$ & $\mathrm{z}$ \\
& & & & \\
\hline
\end{tabular}

Let $\mathrm{S}=\mathrm{M}(\mathrm{G})$ be the parameters and $\mathrm{U}=D_{2}$, dihedral group, be the universal set. We define a fuzzy soft set $f_{s}$ over $U$ by $f_{s}(0)=D_{2}, f_{s}(x)=\{e, b, b a\}, \quad f_{s}(y)=\{a, b\}, f_{s}(z)=\{b\}$.

Then, one can show that $f_{s}$ is fuzzy SU-action on $\mathrm{M}(\mathrm{G})$-ideal of $\mathrm{S}$ over $\mathrm{U}$.

4.2 Example: Consider the near -ring $N=\{0,1,2,3\}$ with the following tables

\begin{tabular}{|l|llll|}
\hline+ & 0 & 1 & 2 & 3 \\
\hline 0 & 0 & 1 & 2 & 3 \\
1 & 1 & 2 & 3 & 0 \\
2 & 2 & 3 & 0 & 1 \\
3 & 3 & 0 & 1 & 2 \\
& & & & \\
\hline
\end{tabular}

\begin{tabular}{|l|llll|}
\hline. & 0 & $\mathrm{x}$ & $\mathrm{y}$ & $\mathrm{z}$ \\
\hline 0 & 0 & 0 & 0 & 0 \\
$\mathrm{x}$ & 0 & 1 & 0 & 1 \\
$\mathrm{y}$ & 0 & 3 & 0 & 3 \\
$\mathrm{z}$ & 0 & 2 & 0 & 2 \\
& & & & \\
\hline
\end{tabular}

Let $\mathrm{S}=\mathrm{M}(\mathrm{G})$ be the set of parameters and $\mathrm{U}=Z^{+}$be the universal set. We define a fuzzy soft set

$f_{s}$ over $\mathrm{U}$ by $f_{s}(0)=\{1,2,3,5,6,7,9,10,11,17\}$

$f_{s}(1)=f_{s}(3)=\{1,3,5,7,9,11\}, f_{s}(2)=\{1,5,7,9,11\}$ 
Since $f_{s}(2 .(3+1)-2.3)=f_{s}(2.1-2.3)=f_{s}(3-3)=f_{s}(0) \nsubseteq f_{s}(1)$

Therefore, $f_{s}$ is not fuzzy SU-action on $\mathrm{M}(\mathrm{G})$-ideal over $\mathrm{U}$. It is known that if $\mathrm{M}(\mathrm{G})$ is a zerosymmetric near-ring, then every $M(G)$-ideal of $S$ is also $M(G)$ - group of $S$. Here, we have an analog for this case.

4.1Theorem: Let $\mathrm{M}(\mathrm{G})$ be a zero- symmetric near-ring. Then, every fuzzy SU-action on $\mathrm{M}(\mathrm{G})$ ideal is fuzzy $\mathrm{SU}$-action on $\mathrm{M}(\mathrm{G})$ - group over $\mathrm{U}$.

Proof: Let $f_{S}$ be an fuzzy SU-action on $\mathrm{M}(\mathrm{G})$-ideal on $\mathrm{S}$ over $\mathrm{U}$. Since $f_{S}(\mathrm{~g}(\mathrm{x}+\mathrm{y})$-gx $) \subseteq f_{S}(\mathrm{y})$, for all $\mathrm{x}, \mathrm{y} \in \mathrm{S}$, and $\mathrm{g} \in \mathrm{M}(\mathrm{G})$, in particular for $\mathrm{x}=0$, it follows that $f_{S}(\mathrm{~g}(0+\mathrm{y})-\mathrm{g} .0)=f_{s}$ (ny$0)=f_{s}(\mathrm{y}) \subseteq f_{s}(\mathrm{y})$. Since the other condition is satisfied by definition-4.1, $f_{s}$ is fuzzy SU-action on $\mathrm{M}(\mathrm{G})$-ideals of $\mathrm{S}$ over $\mathrm{U}$.

4.2Theorem: Let $f_{S}$ be fuzzy SU-action on $\mathrm{M}(\mathrm{G})$-ideal of $\mathrm{S}$ and $f_{T}$ be fuzzy SU-action on $\mathrm{M}(\mathrm{G})$ ideal of T over $\mathrm{U}$. Then $f_{S} \wedge f_{T}$ is fuzzy SU-action on $\mathrm{M}(\mathrm{G})$-ideal of $\mathrm{S} \times \mathrm{T}$ over $\mathrm{U}$.

4.3 Theorem : If $f_{S}$ is fuzzy SU-action on $\mathrm{M}(\mathrm{G})$-ideal of $\mathrm{S}$ and $f_{T}$ be fuzzy $\mathrm{SU}$-action on $\mathrm{M}(\mathrm{G})$ ideal of $\mathrm{T}$ over $\mathrm{U}$, then $f_{S} \times f_{T}$ is fuzzy $\mathrm{SU}$-action on $\mathrm{M}(\mathrm{G})$-ideal over $\mathrm{U} \times \mathrm{U}$.

4.4 Theorem : If $f_{S}$ and $h_{s}$ are two fuzzy SU-action on $\mathrm{M}(\mathrm{G})$ - group of $\mathrm{S}$ over $\mathrm{U}$, then $f_{S} \widetilde{\cap} h_{\mathrm{S}}$ is Fuzzy SU-action on $\mathrm{M}(\mathrm{G})$-ideal over $\mathrm{U}$.

\section{APPLICATION OF FUZZY SU-ACTION ON M(G)- GrouP}

In this section, we give the applications of fuzzy soft image, soft pre-image, lower $\alpha$-inclusion of fuzzy soft sets and $\mathrm{M}(\mathrm{G})$ - group homomorphism with respect to fuzzy SU-action on $\mathrm{M}(\mathrm{G})$ - group and $\mathrm{M}(\mathrm{G})$-ideal.

5.1Theorem: If $f_{S}$ is fuzzy $\mathrm{SU}$-action on $\mathrm{M}(\mathrm{G})$-ideal of $\mathrm{S}$ over $\mathrm{U}$, then $S^{f}=\left\{\mathrm{x} \in \mathrm{S} / f_{S}(\mathrm{x})=f_{S}(0)\right\}$ is a $M(G)$-ideal of $S$.

Proof: It is obvious that $0 \in S^{f}$ we need to show that (i) $\mathrm{x}-\mathrm{y} \in S^{f}$, (ii) s+x-s $\in S^{f}$ and

(iii) $\mathrm{g}(\mathrm{s}+\mathrm{x})$-gs $\in S^{f}$ for all $\mathrm{x}, \mathrm{y} \in S^{f}$ and $\mathrm{g} \in \mathrm{M}(\mathrm{G})$ and $\mathrm{s} \in \mathrm{S}$.

If $\mathrm{x}, \mathrm{y} \in S^{f}$, then $f_{S}(\mathrm{x})=f_{S}(\mathrm{y})=f_{S}(0)$. By proposition-3.1, $f_{S}(0) \subseteq f_{S}(\mathrm{x}-\mathrm{y}), f_{S}(0) \subseteq f_{S}(\mathrm{~s}+\mathrm{x}-\mathrm{s})$, and $f_{S}(0) \subseteq f_{S}(\mathrm{n}(\mathrm{s}+\mathrm{x})$-ns $)$ for all $\mathrm{x}, \mathrm{y} \in S^{f}$ and $\mathrm{g} \in \mathrm{M}(\mathrm{G})$ and $\mathrm{s} \in \mathrm{S}$. Since $f_{S}$ is fuzzy $\mathrm{SU}$-action on $\mathrm{M}(\mathrm{G})$-ideal of $\mathrm{S}$ over $\mathrm{U}$, then for all $\mathrm{x}, \mathrm{y} \in S^{f}$ and $\mathrm{g} \in \mathrm{M}(\mathrm{G})$ and $s \in \mathrm{S}$.

(i) $f_{s}(\mathrm{x}-\mathrm{y}) \subseteq f_{s}(\mathrm{x}) \cup f_{s}(\mathrm{y})=f_{s}(0)$.

(ii) $f_{s}(\mathrm{~s}+\mathrm{x}-\mathrm{s}) \subseteq f_{s}(\mathrm{x})=f_{s}(0)$.

(iii) $f_{s}(\mathrm{~g}(\mathrm{~s}+\mathrm{x})-\mathrm{gs}) \subseteq f_{S}(\mathrm{x})=f_{S}(0)$.

Hence $f_{s}(\mathrm{x}-\mathrm{y})=f_{s}(0), f_{s}(\mathrm{~s}+\mathrm{x}-\mathrm{s})=f_{s}(0)$ and $f_{s}(\mathrm{~g}(\mathrm{~s}+\mathrm{x})$-gs $)=f_{s}(0)$, for all $\mathrm{x}, \mathrm{y} \in S^{f}$ and $\mathrm{g} \in \mathrm{M}(\mathrm{G})$ and $\mathrm{s} \in \mathrm{S}$. Therefore $S^{f}$ is $\mathrm{M}(\mathrm{G})$-ideal of $\mathrm{S}$. 
International Journal of Soft Computing, Mathematics and Control (IJSCMC), Vol. 6, No.1, February 2017

5.2Theorem: Let $f_{s}$ be fuzzy soft set over $\mathrm{U}$ and $\alpha$ be a subset of $\mathrm{U}$ such that $\emptyset \supseteq \alpha \supseteq f_{s}(0)$. If $f_{s}$ is fuzzy $\mathrm{SU}$-action on $\mathrm{M}(\mathrm{G})$-ideal over $\mathrm{U}$, then $f_{S}^{\subseteq \alpha}$ is an $\mathrm{M}(\mathrm{G})$-ideal of $\mathrm{S}$.

Proof: Since $f_{s}(0) \subseteq \alpha$, then $0 \in f_{S}^{\subseteq \alpha}$ and $\emptyset \neq f_{S}^{\subseteq \alpha} \supseteq$ S. Let $\mathrm{x}, \mathrm{y} \in f_{S}^{\subseteq \alpha}$, then $f_{s}(\mathrm{x}) \subseteq \alpha$ and $f_{s}(\mathrm{y}) \subseteq \alpha$. We need to show that

(i) $\mathrm{x}-\mathrm{y} \in f_{S}^{\subseteq \alpha}$

(ii) $\mathrm{s}+\mathrm{x}-\mathrm{s} \in f_{s} \subseteq \alpha$

(iii) $\mathrm{g}(\mathrm{s}+\mathrm{x})-\mathrm{ns} \in f_{s}^{\subseteq \alpha}$ for all $\mathrm{x}, \mathrm{y} \in f_{S}^{\subseteq \alpha}$ and $\mathrm{g} \in \mathrm{M}(\mathrm{G})$ and $\mathrm{s} \in \mathrm{S}$.

Since $f_{s}$ is fuzzy SU-action on $\mathrm{M}(\mathrm{G})$-ideal over $\mathrm{U}$, it follows that

(i) $f_{S}(\mathrm{x}-\mathrm{y}) \subseteq f_{S}(\mathrm{x}) \cup f_{s}(\mathrm{y}) \subseteq \alpha \cup \alpha=\alpha$,

(ii) $f_{s}(\mathrm{~s}+\mathrm{x}-\mathrm{s}) \subseteq f_{s}(\mathrm{x}) \subseteq \alpha$ and

(iii) $f_{s}(\mathrm{~g}(\mathrm{~s}+\mathrm{x})-\mathrm{gs}) \subseteq f_{s}(\mathrm{x}) \subseteq \alpha$. Thus, the proof is completed.

5.3 Theorem : Let $f_{S}$ and $f_{T}$ be fuzzy soft sets over $\mathrm{U}$ and $\chi$ be an $\mathrm{M}(\mathrm{G})$-isomorphism from $\mathrm{S}$ to T.If $f_{s}$ is fuzzy SU-action on $\mathrm{M}(\mathrm{G})$-ideal of $\mathrm{S}$ over $\mathrm{U}$, then $\chi\left(f_{s}\right)$ is fuzzy SU-action on $\mathrm{M}(\mathrm{G})$ ideal of T over $\mathrm{U}$.

Proof: Let $\delta_{1}, \delta_{2}$ and $\mathrm{g} \in \mathrm{M}(\mathrm{G})$. Since $\chi$ is surjective, there exists $s_{1}, s_{2} \in \mathrm{S}$ such that $\chi\left(s_{1}\right)=\delta_{1}$ and $\chi\left(s_{2}\right)=\delta_{2}$. Then

$$
\begin{aligned}
\left(\chi f_{s}\right)\left(\delta_{1}-\delta_{2}\right) & =\cup\left\{f_{s}(\mathrm{~s}) / \mathrm{s} \in \mathrm{S}, \chi(\mathrm{s})=\delta_{1}-\delta_{2}\right\} \\
& =\cup\left\{f_{s}(\mathrm{~s}) / \mathrm{s} \in \mathrm{S}, \mathrm{s}=\chi^{-1}\left(\delta_{1}-\delta_{2}\right)\right\} \\
& =\cup\left\{f_{s}(\mathrm{~s}) / \mathrm{s} \in \mathrm{S}, \mathrm{s}=\chi^{-1}\left(\chi\left(s_{1}-s_{2}\right)\right)=s_{1}-s_{2}\right\} \\
& =\cup\left\{f_{s}\left(s_{1}-s_{2}\right) / s_{i} \in \mathrm{S}, \chi\left(s_{i}\right)=\delta_{i}, i=1,2, \ldots\right\} \\
& \left.\subseteq \cup\left\{f_{s}\left(s_{1}\right) \cup f_{s}\left(s_{2}\right)\right) / s_{i} \in \mathrm{S}, \chi\left(s_{i}\right)=\delta_{i}, i=1,2, \ldots\right\} \\
& =\left(\left\{\cup\left\{f_{s}\left(s_{1}\right) / s_{1} \in \mathrm{S}, \chi\left(s_{1}\right)=\delta_{1}\right\}\right\} \cup\left\{\cup\left\{f_{s}\left(s_{2}\right) / s_{2} \in \mathrm{S}, \chi\left(s_{2}\right)=\delta_{2}\right\}\right\}\right) \\
& =\left(\chi\left(f_{s}\right)\right)\left(\delta_{1}\right) \cup\left(\chi\left(f_{s}\right)\right)\left(\delta_{2}\right)
\end{aligned}
$$

$$
\text { Also } \begin{aligned}
\left(\chi f_{s}\right)\left(\delta_{1}+\delta_{2}-\delta_{1}\right) & =\cup\left\{f_{s}(\mathrm{~s}) / \mathrm{s} \in \mathrm{S}, \chi(\mathrm{s})=\delta_{1}+\delta_{2}-\delta_{1}\right\} \\
& =\cup\left\{f_{s}(\mathrm{~s}) / \mathrm{s} \in \mathrm{S}, \mathrm{s}=\chi^{-1}\left(\delta_{1}+\delta_{2}-\delta_{1}\right)\right\} \\
& =\cup\left\{f_{s}(\mathrm{~s}) / \mathrm{s} \in \mathrm{S}, \mathrm{s}=\chi^{-1}\left(\chi\left(s_{1}+s_{2}-s_{1}\right)\right)=s_{1}+s_{2}-s_{1}\right\} \\
& =\cup\left\{f_{s}\left(s_{1}+s_{2}-s_{1}\right) / s_{i} \in \mathrm{S}, \chi\left(s_{i}\right)=\delta_{i}, i=1,2, \ldots\right\} \\
& \subseteq \cup\left\{f_{s}\left(s_{2}\right) / s_{2} \in \mathrm{S}, \chi\left(s_{2}\right)=\delta_{2}\right\} \\
& =\left(\chi\left(f_{s}\right)\right)\left(\delta_{2}\right)
\end{aligned}
$$

Furthermore, $\left(\chi f_{s}\right)\left(\mathrm{g}\left(\delta_{1}+\delta_{2}\right)-\mathrm{g} \delta_{1}\right)=U\left\{f_{s}(\mathrm{~s}) / \mathrm{s} \in \mathrm{S}, \chi(\mathrm{s})=\mathrm{g}\left(\delta_{1}+\delta_{2}\right)-\mathrm{g} \delta_{1}\right\}$

$$
\begin{aligned}
& =\cup\left\{f_{s}(\mathrm{~s}) / \mathrm{s} \in \mathrm{S}, \mathrm{s}=\chi^{-1}\left(\mathrm{~g}\left(\delta_{1}+\delta_{2}\right)-\mathrm{g} \delta_{1}\right)\right\} \\
& =\cup\left\{f_{s}(\mathrm{~s}) / \mathrm{s} \in \mathrm{S}, \mathrm{s}=\mathrm{g}\left(s_{1}+s_{2}\right)-\mathrm{g} s_{1}\right\} \\
& \left.=\cup\left\{f_{s}\left(g+s_{2}\right)-\mathrm{g} s_{1}\right) / s_{i} \in \mathrm{S}, \chi\left(s_{i}\right)=\delta_{i}, i=1,2, \ldots\right\} \\
& \subseteq \cup\left\{f_{s}\left(s_{2}\right) / s_{2} \in \mathrm{S}, \chi\left(s_{2}\right)=\delta_{2}\right\} \\
& =\left(\chi\left(f_{s}\right)\right)\left(\delta_{2}\right) .
\end{aligned}
$$

Hence $\chi\left(f_{s}\right)$ is fuzzy SU-action on $\mathrm{M}(\mathrm{G})$-ideal of T over $\mathrm{U}$. 
5.4Theorem: Let $f_{S}$ and $f_{T}$ be fuzzy soft sets over $\mathrm{U}$ and $\chi$ be an $\mathrm{M}(\mathrm{G})$--isomorphism from $\mathrm{S}$ to T. If $f_{T}$ is fuzzy SU-action on $\mathrm{M}(\mathrm{G})$-ideal of $\mathrm{T}$ over $\mathrm{U}$, then $\chi^{-1}\left(f_{T}\right)$ is fuzzy SU-action on $\mathrm{M}(\mathrm{G})$-ideal of $\mathrm{S}$ over $\mathrm{U}$.

Proof: Let $s_{1}, s_{2} \in \mathrm{S}$ and $\mathrm{g} \in \mathrm{M}(\mathrm{G})$. Then

$$
\begin{aligned}
\left(\chi^{-1}\left(f_{T}\right)\right)\left(s_{1}-s_{2}\right)= & f_{T}\left(\chi\left(s_{1}-s_{2}\right)\right) \\
& =f_{T}\left(\chi\left(s_{1}\right)-\chi\left(s_{2}\right)\right) \\
& \subseteq f_{T}\left(\chi\left(s_{1}\right)\right) \cup f_{T}\left(\chi\left(s_{2}\right)\right) \\
& =\left(\chi^{-1}\left(f_{T}\right)\right)\left(s_{1}\right) \cup\left(\chi^{-1}\left(f_{T}\right)\right)\left(s_{2}\right) .
\end{aligned}
$$

Also $\left(\chi^{-1}\left(f_{T}\right)\right)\left(s_{1}+s_{2}-s_{1}\right)=f_{T}\left(\chi\left(s_{1}+s_{2}-s_{1}\right)\right)$

$$
\begin{aligned}
& =f_{T}\left(\chi\left(s_{1}\right)+\chi\left(s_{2}\right)-\chi\left(s_{1}\right)\right) \\
& \subseteq f_{T}\left(\chi\left(s_{2}\right)\right)=\left(\chi^{-1}\left(f_{T}\right)\right)\left(s_{2}\right)
\end{aligned}
$$

Furthermore, $\left(\chi^{-1}\left(f_{T}\right)\right)\left(\mathrm{g}\left(s_{1}+s_{2}\right)-\mathrm{g} s_{1}\right)=f_{T}\left(\chi\left(\mathrm{g}\left(s_{1}+s_{2}\right)-\mathrm{g} s_{1}\right)\right)$

$$
\begin{aligned}
& =f_{T}\left(\mathrm{~g}\left(\chi\left(s_{1}\right)+\chi\left(s_{2}\right)\right)-\mathrm{g} \chi\left(s_{1}\right)\right) \\
& \subseteq f_{T}\left(\chi\left(s_{2}\right)\right)=\left(\chi^{-1}\left(f_{T}\right)\right)\left(s_{2}\right)
\end{aligned}
$$

Hence, $\left(\chi^{-1}\left(f_{T}\right)\right)$ is fuzzy SU-action on $\mathrm{M}(\mathrm{G})$-ideal of $\mathrm{S}$ over $\mathrm{U}$.

\section{CONCLUSION:}

In this paper, we have defined a new type of N-module action on a fuzzy soft set, called fuzzy SU-action on $\mathrm{M}(\mathrm{G})$ - group by using the soft sets. This new concept picks up the soft set theory, fuzzy theory and $M(G)$ - group theory together and therefore, it is very functional for obtaining results in the mean of $\mathrm{M}(\mathrm{G})$ - group structure. Based on this definition, we have introduced the concept of fuzzy SU-action on $\mathrm{M}(\mathrm{G})$-ideal. We have investigated these notions with respect to soft image, soft pre-image and lower $\alpha$-inclusion of soft sets. Finally, we give some application of fuzzy SU-action on $\mathrm{M}(\mathrm{G})$-ideal to $\mathrm{M}(\mathrm{G})$ - group theory. To extend this study, one can further study the other algebraic structures such as different algebra in view of their SU-actions.

\section{ACKNOWLEDGEMENT:}

The authors are highly grateful to the referees for their valuable comments and suggestions for improving papers.

\section{REFERENCES}

[1] Acar U., Koyuncu F., Tanay B., Soft sets and soft rings, Comput. Math. Appl., 59(2010), 3458-3463.

[2] Aktas. H., C. agman N., ` Soft sets and soft groups, Inform. Sci., 177(2007), 2726-2735.

[3] Ali M.I.,Feng F., Liu X., Min W.K., Shabir M., On some new operations in soft set theory, Comput. Math. Appl., 57(2009), 1547-1553.

[4] Atagun A.O., Sezgin A., Soft substructures of rings, fields and modules, Comput. Math. Appl., 61(3)(2011), 592-601.

[5] Babitha K.V., Sunil J.J., Soft set relations and functions, Comput. Math. Appl., 60(7)(2010), 1840-1849. 
[6] Cagman N., Enginoglu S.,Soft matrix theory and its decision making, Comput. Math. Appl., 59(2010), 3308-3314.

[7] Cagman N, Engino ` glu S., ` Soft set theory and uni-int decision making, Eur. J. Oper. Res., 207(2010), 848-855.

[8] Cagman N., C, 1tak F., Aktas, H., ' Soft int-groups and its applications to group theory, Neural Comput. Appl., DOI: 10.1007/s00521-011-0752-x.

[9] Cagman N., Sezgin A., Atag `un A.O., " Soft uni-groups and its applications to group theory, (submitted).

[10] Cagman N., Sezgin A., Atag ` un A.O., ${ }^{\sim} \alpha$-inclusions and their applications to group theory, (submitted). ${ }^{\smile}$

[11] F. Feng, W. Pedrycz, On scalar products and decomposition theorems of fuzzy soft sets, Journal of Multivalued Logic and Soft Computing, 2015, 25(1), 45-80.

[12] F. Feng, J. Cho, W. Pedrycz, H. Fujita, T. Herawan, Soft set based association rule mining, Knowledge-Based Systems, 111 (2016), 268-282

[13] Feng F., Li C., Davvaz B., Ali M.I., Soft sets combined with fuzzy sets and rough sets: a tentative approach, Soft Comput., 14(6)(2010), 899-911.

[14] Jun Y.B., Soft BCK/BCI-algebras, Comput. Math. Appl., 56(2008), 1408 -1413.

[15] Jun Y.B., Park C.H., Applications of soft sets in ideal theory of BCK/ BCI-algebras, Inform. Sci., 178(2008), 2466-2475.

[16] Jun Y.B., Lee K.J., Zhan J., Soft p-ideals of soft BCI-algebras, Comput. Math. Appl., 58(2009), 2060-2068.

[17] Jun Y.B., Lee K.J., Park C.H., Soft set theory applied to ideals in d-algebras, Comput. Math. Appl., 57(3)(2009), 367-378.

[18] Kazancı O., Yılmaz S,., Yamak S., Soft sets and soft BCH-algebras, Hacet. J. Math. Stat., 39(2)(2010), 205-217.

[19] Maji P.K., Biswas R., Roy A.R., Soft set theory, Comput. Math. Appl., 45(2003), 555-562.

[20] Maji P.K., Roy A.R., Biswas R., An application of soft sets in a decision making problem, Comput. Math. Appl., 44(2002), 1077-1083.

[21] Majumdar P., Samanta S.K., On soft mappings, Comput. Math. Appl., 60 (9)(2010), 26662672.

[22] Molodtsov D., Soft set theory-first results, Comput. Math. Appl., 37(1999), 19-31. 
International Journal of Soft Computing, Mathematics and Control (IJSCMC), Vol. 6, No.1, February 2017

[23] Molodtsov D.A., Leonov V.Yu., Kovkov D.V., Soft sets technique and its application, Nechetkie Sistemy i Myagkie Vychisleniya, 1(1)(2006), 8-39.

[24] Pilz G., Near-rings, North Holland Publishing Company, Amsterdam-New York-Oxford, 1983.

[25] Sezgin A., Atagun A.O., Ayg " un E., " A note on soft near-rings and idealistic soft nearrings, Filomat., 25(1)(2011), 53-68.

[26] Sezgin A., Atagun A.O., “ On operations of soft sets, Comput. Math. Appl., 61(5)(2011), $1457-1467$.

[27] Wendt G., On Zero Divisors in Near-Rings, Int. J. Algebra, 3(1)(2009), 21-32.

[28] Zhan J., Jun Y.B., Soft BL-algebras based on fuzzy sets, Comput. Math. Appl., 59(6)(2010), 2037-2046.

[29] Zou Y., Xiao Z., Data analysis approaches of soft sets under incomplete information, KnowlBased Syst., 21(2008), 941-945.

[30] J. Zhan, B. Yu, V. Fotea, Characterizations of two kinds of hemi rings based on probability spaces, Soft Comput, 20 (2016), 637-648. 